\title{
Universiteit
}

Leiden

The Netherlands

\section{Imaging of vortex configurations in thin films by scanning-tunneling microscopy}

Baarle, G.J.C. van; Troyanovski, A.M.; Nishizaki, T.; Kes, P.H.; Aarts, J.

\section{Citation}

Baarle, G. J. C. van, Troyanovski, A. M., Nishizaki, T., Kes, P. H., \& Aarts, J. (2003). Imaging of vortex configurations in thin films by scanning-tunneling microscopy. Applied Physics Letters, 82(7), 1081-1083. doi:10.1063/1.1554481

Version: $\quad$ Not Applicable (or Unknown)

License: $\quad$ Leiden University Non-exclusive license

Downloaded from: https://hdl.handle.net/1887/45200

Note: To cite this publication please use the final published version (if applicable). 


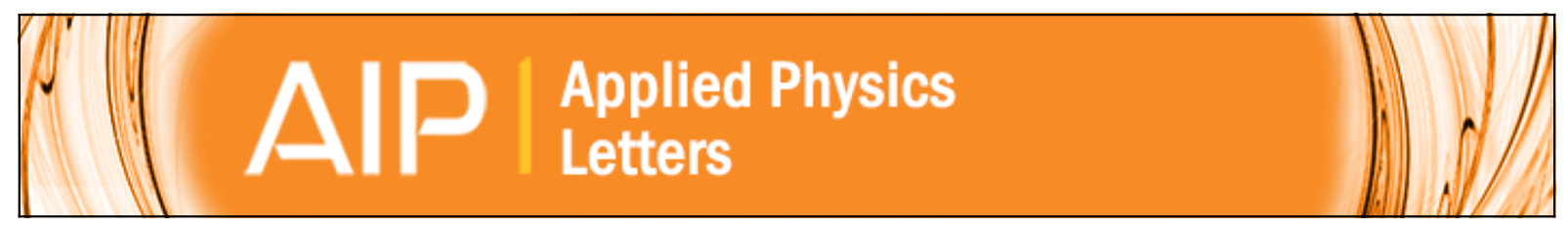

\section{Imaging of vortex configurations in thin films by scanning-tunneling microscopy}

G. J. C. van Baarle, A. M. Troianovski, T. Nishizaki, P. H. Kes, and J. Aarts

Citation: Applied Physics Letters 82, 1081 (2003); doi: 10.1063/1.1554481

View online: http://dx.doi.org/10.1063/1.1554481

View Table of Contents: http://scitation.aip.org/content/aip/journal/apl/82/7?ver=pdfcov

Published by the AIP Publishing

\section{Articles you may be interested in}

Lateral imaging of the superconducting vortex lattice using Doppler-modulated scanning tunneling microscopy Appl. Phys. Lett. 99, 192505 (2011); 10.1063/1.3659412

Peak effect and dynamic melting transitions of driven vortex system in weakly disordered Josephson junction arrays

J. Appl. Phys. 108, 103911 (2010); 10.1063/1.3514140

Electronic Transport in the Low-Temperature Liquid Phase of a Thin Amorphous Film without the Edge Effects AIP Conf. Proc. 850, 969 (2006); 10.1063/1.2355028

Temperature dependence of vortex configuration by honeycomb hole arrays in a superconducting $\mathrm{Nb}$ film J. Appl. Phys. 97, 10B102 (2005); 10.1063/1.1849511

Nature of critical current and coherent phenomena in granular MoN $x$ thin films

Low Temp. Phys. 26, 881 (2000); 10.1063/1.1334438

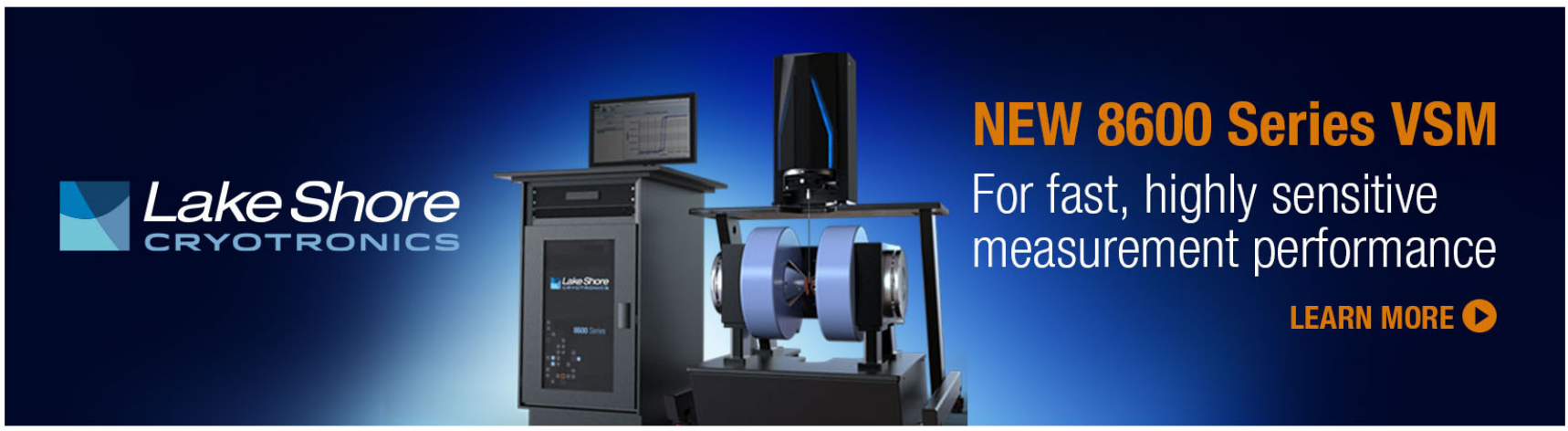




\title{
Imaging of vortex configurations in thin films by scanning-tunneling microscopy
}

\author{
G. J. C. van Baarle, ${ }^{\text {a) }}$ A. M. Troianovski, ${ }^{\text {b) }}$ T. Nishizaki, ${ }^{\text {c) }}$ P. H. Kes, and J. Aarts \\ Kamerlingh Onnes Laboratory, Leiden University, P.O. Box 9504, 2300RA Leiden, The Netherlands
}

(Received 22 October 2002; accepted 2 January 2003)

\begin{abstract}
We report on imaging of vortices in thin superconducting films using surface passivation with an ultrathin Au layer. This allows investigation of surfaces that oxidize easily, as well as the mounting of samples in air. We studied vortex configurations in a material with weak vortex pinning $\left(a-\mathrm{Mo}_{2.7} \mathrm{Ge}\right)$ and a strongly pinning material $(\mathrm{NbN})$ at $4.2 \mathrm{~K}$ in magnetic fields up to $1.4 \mathrm{~T}$. In $a-\mathrm{Mo}_{2.7} \mathrm{Ge}$, we observe a well-ordered hexagonal lattice, with local defects beginning to appear around $1.0 \mathrm{~T}$. In NbN, the vortex lattice is fully disordered. (c) 2003 American Institute of Physics. [DOI: $10.1063 / 1.1554481]$
\end{abstract}

Scanning tunneling microscopy (STM) is a useful technique for imaging vortex structures in superconductors. One of the principal advantages in comparison with other imaging tools, such as magneto-optics, Bitter decoration, or magnetic force microscopy, is that STM measures the electronic density of states instead of the magnetic flux density, thus being sensitive on the scale of the superconducting coherence length $\xi$, rather than the magnetic penetration depth $\lambda$. This opens a much larger magnetic field region for investigations, in particular the field region where the vortexvortex spacing is smaller than $\lambda$. However, due to the requirements of having a clean, almost atomically flat surface, vortices have been observed by STM mainly in four different single-crystalline materials: $\mathrm{NbSe}_{2},{ }^{1} \mathrm{YBa}_{2} \mathrm{Cu}_{3} \mathrm{O}_{7-\delta},{ }^{2}$ $\mathrm{Bi}_{2} \mathrm{Sr}_{2} \mathrm{CaCu}_{2} \mathrm{O}_{8+\delta},{ }^{3}$ and $(\mathrm{Lu} / \mathrm{Y}) \mathrm{Ni}_{2} \mathrm{~B}_{2} \mathrm{C},{ }^{4,5}$ while in thin films we know of only one report on observing vortex signatures. ${ }^{6}$

In this letter, we report on the result of a passivation technique to overcome such major obstacles as surface roughness and oxidation, which make tunneling and scanning impossible. We use it to investigate the vortex structures in two different superconductors, $a-\mathrm{Mo}_{2.7} \mathrm{Ge}$ ( = amorphous $\mathrm{Mo}_{70} \mathrm{Ge}_{30}$ ) and $\mathrm{NbN}$, at $4.2 \mathrm{~K}$ and up to $B$ $=1.4 \mathrm{~T}$. Both materials can be prepared in thin film form; $a-\mathrm{Mo}_{2.7} \mathrm{Ge}$ has a superconducting transition temperature $T_{c}$ of $6.5 \mathrm{~K}$ and an upper critical field $B_{c 2}$ at $4.2 \mathrm{~K}$ of $6 \mathrm{~T}$. In addition, it shows moderate intrinsic pinning strength, basically due to the absence of grain boundaries; for a typical film thickness of $50 \mathrm{~nm}$ at intermediate fields and low temperatures, the critical current density $J_{c}$ for onset of vortex motion is of the order of $10^{6} \mathrm{~A} / \mathrm{m}^{2}{ }^{7}$ In strongly pinning $\mathrm{NbN}$ with a $T_{c}$ of $11 \mathrm{~K}$ and a $B_{c 2}$ at $4.2 \mathrm{~K}$ of about $8.8 \mathrm{~T}, J_{c}$ is at least two orders of magnitude higher. ${ }^{8}$

For amorphous films, spectroscopic imaging is feasible because of the very flat surface characteristics. However, even a small amount of oxidation is detrimental to mapping

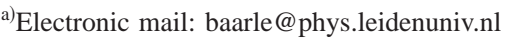

${ }^{\mathrm{b})}$ Present address: Kapitza Institute for Physical Problems, Russian Academy of Sciences, Kosygina 2, Moscow, 119334, Russia.

${ }^{c}$ Present address: Institute for Materials Research, Tohoku University, Sendai 980-8577, Japan.
}

the superconducting gap, which is the way to determine vortex core positions. To prevent this, we cap the amorphous film with a very thin Au film. It turns out that sputtering even $3 \mathrm{~nm}$ of Au on $a-\mathrm{Mo}_{2.7} \mathrm{Ge}$ is enough to form a closed layer, which still allows one to perform gap spectroscopy due to the proximity effect. For crystalline films such as NbN, oxidation also has to be prevented, but here, a capping Au layer does not close so easily. For a 50-nm NbN layer, passivation by a Au layer is possible, ${ }^{9}$ but better results are obtained by depositing a thin layer $(20 \mathrm{~nm})$ of $a-\mathrm{Mo}_{2.7} \mathrm{Ge}$ followed by the Au capping layer. This results in a flatter surface than when $\mathrm{Au}$ is directly deposited on $\mathrm{NbN}$, and it has a more uniform thickness, which is an advantage for the proximity effect. The important point to realize is that the vortex positions as measured on the Au surface are still dictated by the pinning of the vortices in the $\mathrm{NbN}$ layer, since the pinning by the $a-\mathrm{Mo}_{2.7} \mathrm{Ge}$ layer is much too weak to influence the vortex configuration.

The 50-nm-thick $a-\mathrm{Mo}_{2.7} \mathrm{Ge}$ films were rf sputtered from a composite $\mathrm{Mo} / \mathrm{Ge}$ target at room temperature in an Ar atmosphere on a Si substrate. Atomic force microscopy (AFM) on such a film showed a flat surface over the scan range of $0.5 \mu \mathrm{m}$ within the resolution of our AFM measurement (0.4 $\mathrm{nm})$. Next, we deposited the Au capping layer immediately after depositing the $a-\mathrm{Mo}_{2.7} \mathrm{Ge}$ by first sputtering about 3-nm $\mathrm{Au}$ in $\mathrm{Ar}(100 \%)$ atmosphere to obtain a closed Au layer, followed by another $3 \mathrm{~nm}$ in $\mathrm{Ar} / \mathrm{O}_{2}$ (95\%/5\% partial pressure) to obtain a flat Au surface consisting of small grains. Highest resolution is achieved having Au grains as small as possible, which is promoted by the $\mathrm{O}_{2}$ addition. The 50-nmthick $\mathrm{NbN}$ samples were reactively rf-sputtered at room temperature from a $\mathrm{Nb}$ target in an $\mathrm{Ar} / \mathrm{N}_{2}$ atmosphere $(95 \% / 5 \%$ partial pressure). This was followed by a 20-nm layer of $a-\mathrm{Mo}_{2.7} \mathrm{Ge}$ and a $\mathrm{Au}$ layer as for the $a-\mathrm{Mo}_{2.7} \mathrm{Ge}$ films. After sputtering, the samples were taken out of the vacuum and mounted on the scanner of the STM. During this procedure, which takes about half an hour, the samples were exposed to air.

Examples of the topography as measured by STM can be found in Refs. 9 and 10. The surface shows grains with a size of $10 \mathrm{~nm}$ and a maximum height variation of $1 \mathrm{~nm}$ over a scan length of $400 \mathrm{~nm}$. The STM measurements were per- 

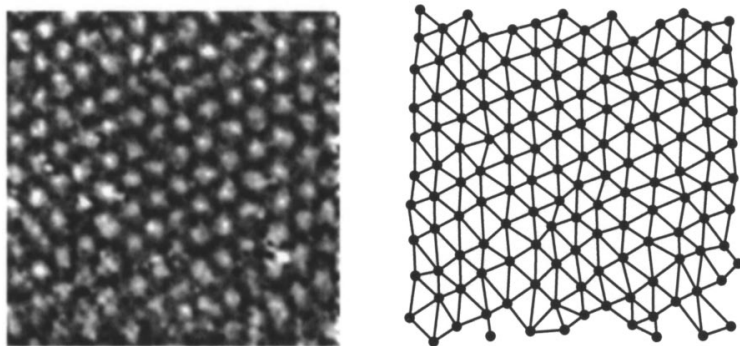

(a) MoGe

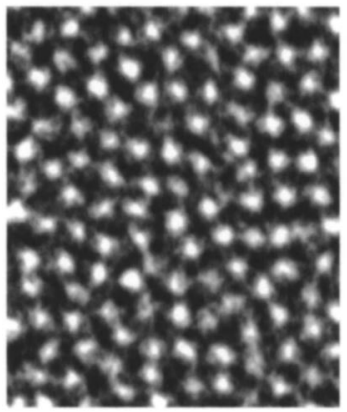

(b) $\mathrm{NbN}$ (c)

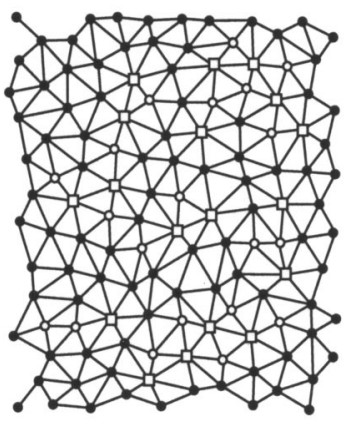

(d)

FIG. 1. Vortex configurations as measured in $a-\mathrm{Mo}_{2.7} \mathrm{Ge}$ and $\mathrm{NbN}$ at $4.2 \mathrm{~K}$ and magnetic fields of 0.5 and $0.8 \mathrm{~T}$, respectively. Scan ranges are 704 $\times 704 \mathrm{~nm}^{2}$ and $510 \times 680 \mathrm{~nm}^{2}$, respectively. On the right, the corresponding triangulations are shown. Black circles correspond to vortices with coordination number $z=6$, open circles have $z<6$, while the open squares mark vortices with $z>6$.

formed in the same setup as mentioned in Ref. 11, and the STM itself is directly immersed in the liquid helium; all data shown are acquired at $4.2 \mathrm{~K}$.

The vortex imaging is done by acquiring full $I-V$ tunneling spectra simultaneously with the topography measurement (constant current mode): at each point, after stabilizing the tip height, the feedback is switched off and an $I-V$ curve is measured. From these $I-V$ curves, we evaluate the ratio of the zero-bias and high-bias (larger than the superconducting gap energy $\Delta$ ) slopes, providing information about the proximity-induced, local quasiparticle density of states, $N_{S^{\prime}}(E, r)$, where $E$ is the energy of the quasiparticles and $r$ is the position of the tip on the sample. On choosing a resolution of $128 \times 128$ points per image the acquisition time is about $1 \mathrm{~h}$. For all vortex images shown, except otherwise mentioned, we applied some basic image processing to the images (smoothing and balancing gray scale).

In Figs. 1(a) and 1(b) we show results obtained on $a-\mathrm{Mo}_{2.7} \mathrm{Ge}$ in a field of $0.5 \mathrm{~T}$ and on $\mathrm{NbN}$ in a field of $0.8 \mathrm{~T}$, which corresponds to the same reduced field $b=B / B_{c 2}$ $=0.09$ for both samples. These results demonstrate that we are able to clearly image vortex structures on both films. The vortex cores are mostly well defined, although there is variation in the brightness both within the core and comparing different cores. This is due mainly to the grain structure of the Au layer: $N_{S^{\prime}}(E, r)$ is constant in a grain, ${ }^{12}$ but can vary due to inelastic scattering at grain boundaries. The scan range is chosen such that, for both samples, around 130 vortices are imaged. The vortex density $d$ of $258 \pm 20 \mu \mathrm{m}^{-2}$ $\left(a-\mathrm{Mo}_{2.7} \mathrm{Ge}\right)$ and $389 \pm 20 \mu \mathrm{m}^{-2}(\mathrm{NbN})$ corresponds well to the expected value, $d=B / \Phi_{0}=242$ and $385 \mu \mathrm{m}^{-2}$, respectively (with $\Phi_{0}$ the flux quantum).
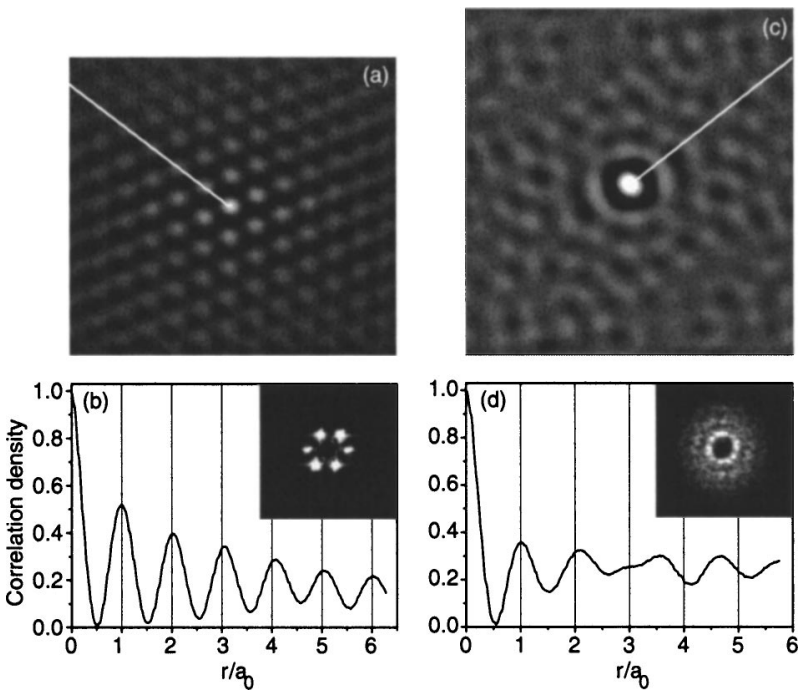

FIG. 2. Autocorrelation data $(a$ and $c$ ) of the images shown in Fig. 1. Panels $b$ and $d$ show a cross section along the white lines in panels $a$ and $c$. The horizontal axis is in units of the vortex-vortex distance $a_{0}$. The insets show the 2D Fourier transforms.

The vortex lattice (VL) configuration in both films is quite different. A quick way to demonstrate this is to perform a triangulation analysis to find the number of nearest neighbors for each vortex, which should be six for the perfect triangular lattice. The triangulation results given in Figs. 1(c) and 1 (d) show that the VL in $a-\mathrm{Mo}_{2.7} \mathrm{Ge}$ is without any defects, while in the case of $\mathrm{NbN}$, a large number of vortices $(36 \%)$ has five- or sevenfold coordination.

For a more quantitative analysis of the correlation of vortex positions, we generated two-dimensional (2D) maps of the autocorrelation function (with distances $r$ now scaled on $a_{0}$, the vortex-vortex distance defined from the peaks in the autocorrelation graphs), shown in Fig. 2. These maps directly yield an estimate for the VL correlation length $R_{c}$, in contrast to the often-presented 2D Fourier transforms [shown in the insert of Figs. 2(b) and 2(d)] where this information is in the width of the peaks. From the pictures, it is evident that in $a-\mathrm{Mo}_{2.7} \mathrm{Ge}$ the VL has both high translational (bond length) and rotational (bond orientation) correlation, with a lower bound of $R_{c} \approx 6 a_{0}$ set by the limited amount of vortices. For $\mathrm{NbN}$, we find $R_{c} \approx 1.5 a_{0}$ and almost no orientational correlation. The gray features that still can be seen in Fig. 2(c), the small variations of correlation above $2 r / a_{0}$, and the nonuniformity of the ring in the Fourier transform in Fig. 2(d) are due to the limited amount of vortices on which to do these statistics.

Next, we consider the field dependence of the correlations. No qualitative changes were found in the VL structure of the $\mathrm{NbN}$ films up to $1.4 \mathrm{~T}$, the highest magnetic field measured. In the whole field regime, we therefore essentially find $R_{c} \approx(1-2) a_{0}$. This confirms on a local scale the conclusion from pinning force measurements, that in the field regime below $b \approx 0.15$, grain boundary pinning is more important than the elastic interactions between vortices, leading to isolated vortex behavior. ${ }^{13}$ For $a-\mathrm{Mo}_{2.7} \mathrm{Ge}$, we show additional data of measurements at 0.07, 0.3, and $1.0 \mathrm{~T}$ in Fig. 3. In the lowest fields, we have the problem that the scan range, and therefore the total amount of vortices that can be imaged, is limited. However, inspection of Fig. 3(a) reveals several 



FIG. 3. Images of $a-\mathrm{Mo}_{2.7} \mathrm{Ge}$ vortex lattices for various flux densities. Magnetic field and scan size are respectively: (a) $0.07 \mathrm{~T}, 700 \times 858 \mathrm{~nm}^{2}$, (b) 0.3 $\mathrm{T}, 700 \times 858 \mathrm{~nm}^{2}$, (c) and (d) $1.0 \mathrm{~T}, 484 \times 484 \mathrm{~nm}^{2}$. In panel (a) the triangulation is represented by the dashed lines. The lattice deformation in panel (b) and the defects appearing in panel (d) are indicated.

defects. For higher fields, up to $1.0 \mathrm{~T}$, we always obtained images without any defects, although the lattice as a whole may show deformations, visible in the data at $B=0.3 \mathrm{~T}$ [Fig. 3(b)]. Since the fast-scan direction is horizontal, this deformation is a real property of the lattice rather than an artifact of the measurement. We also observe rotations of the VL after changing the magnetic field and measuring again at the same spot: panels (a) and (b) of Fig. 3 are subsequently measured on the same location on the film. We propose that these observations are due to the presence of large domains of different orientation that change in size and orientation after a field step. This emphasizes once more the collective nature of the forces in VLs of amorphous superconductors, due to the weak random nature of the pinning.

For the $1.0 \mathrm{~T}$ data, we used a convolution filter with a kernel of size $a_{0}^{2}$, since at these magnetic fields, the contrast becomes poor due to the overall suppression of the superconducting gap. Before measuring these images, we waited for $1.5 \mathrm{~h}$ to let the VL relax after the field was set to $1.0 \mathrm{~T}$. Subsequently, we acquired 10 consecutive images on the same location of the film, each having an acquisition time of around $90 \mathrm{~min}$. From this set of images, two subsequent images showed a lattice without defects, while all the others showed one or more defects on different locations in the lattice. Images without and with a defect are shown in Figs. 3(c) and 3(d). These apparently quite mobile defects indicate spontaneous nucleation of defects, which may be the first signal of the onset of melting at higher fields. ${ }^{14}$

To summarize, we have developed a technique which makes imaging vortex configurations in superconducting thin films possible in magnetic fields that were not accessible before. We applied the technique to films of $a-\mathrm{Mo}_{2.7} \mathrm{Ge}$ and $\mathrm{NbN}$, resulting in clear images of their vortex configurations. Measurements on $a-\mathrm{Mo}_{2.7} \mathrm{Ge}$, a weak pinning material, show a triangular vortex lattice with defects appearing around 1.0 T. For $\mathrm{NbN}$, a strong pinning material, the lattice is fully disordered. The technique can, in principle, be used to observe vortex configurations in any type-II superconductor on which Au can be grown in a smooth layer (possibly with the help of $a-\mathrm{Mo}_{2.7} \mathrm{Ge}$ ). This opens a way to study the microscopic behavior of the vortices, for example, at phase transitions, and can produce information on artificially structured samples.

This work is part of the research program of the Stichting voor Fundamenteel Onder-zoek der Materie (FOM), which is financially supported by the Nederlandse Organisatie voor Wetenschappelijk Onderzoek (NWO). One of the authors (T.N.) acknowledges support from NWO and the Japanese Society for Promotion of Science (JSPS).

${ }^{1}$ H. F. Hess, R. B. Robinson, R. C. Dynes, J. M. Valles, Jr., and J. V. Waszczak, Phys. Rev. Lett. 62, 214 (1989).

${ }^{2}$ I. Maggio-Aprile, Ch. Renner, A. Erb, E. Walker, and Ø. Fischer, Phys. Rev. Lett. 75, 2754 (1995).

${ }^{3}$ Ch. Renner, B. Revaz, K. Kadowaki, I. Maggio-Aprile, and $\varnothing$. Fischer, Phys. Rev. Lett. 80, 3606 (1998).

${ }^{4}$ Y. de Wilde, M. Iavarone, U. Welp, V. Metlushko, A. E. Koshelev, I. Aranson, G. W. Crabtree, and P. C. Canfield, Phys. Rev. Lett. 78, 4273 (1997).

${ }^{5}$ H. Sakata, M. Oosawa, K. Matsuba, N. Nishida, H. Takeya, and K. Hirata, Phys. Rev. Lett. 84, 1583 (2000).

${ }^{6}$ S. Kashiwaya, M. Koyanagi, and A. Shoji, Appl. Phys. Lett. 61, 1847 (1992).

${ }^{7}$ R. Besseling (private communication).

${ }^{8}$ A. Pruymboom, W. H. B. Hoondert, H. W. Zandbergen, and P. H. Kes, Jpn. J. Appl. Phys. 26, Suppl. 26-3, 1529 (1987)

${ }^{9}$ T. Nishizaki, A. M. Troyanovski, G. J. C. van Baarle, P. H. Kes, and J. Aarts, (in press).

${ }^{10}$ G. J. C. van Baarle, A. M. Troianovski, P. H. Kes, and J. Aarts, Physica C 369, 335 (2002).

${ }^{11}$ A. Troyanovskii, J. Aarts, and P. H. Kes, Nature (London) 399, 665 (1999).

${ }^{12}$ A. D. Truscott, R. C. Dynes, and L. F. Schneemeyer, Phys. Rev. Lett. 83, 1014 (1999).

${ }^{13}$ A. Pruymboom, W. H. B. Hoondert, H. W. Zandbergen, and P. H. Kes, Jpn. J. Appl. Phys. 26, Suppl. 26-3, 1531 (1987).

${ }^{14}$ P. Berghuis and P. H. Kes, Phys. Rev. B 47, 262 (1993). 